\title{
Spin-Orbit Coupling and Electronic Correlations in $\mathrm{Sr}_{2} \mathrm{RuO}_{4}$
}

\author{
Minjae Kim, ${ }^{1,2}$, * Jernej Mravlje, ${ }^{3}$ Michel Ferrero, ${ }^{1,2}$ Olivier Parcollet, ${ }^{4,2}$ and Antoine Georges ${ }^{2,5,1,6}$ \\ ${ }^{1}$ Centre de Physique Théorique, École Polytechnique, \\ CNRS, Université Paris-Saclay, 91128 Palaiseau, France \\ ${ }^{2}$ Collège de France, 11 place Marcelin Berthelot, 75005 Paris, France \\ ${ }^{3}$ Jožef Stefan Institute, Jamova 39, Ljubljana, Slovenia \\ ${ }^{4}$ Institut de Physique Théorique (IPhT), CEA, CNRS, 91191 Gif-sur-Yvette, France \\ ${ }^{5}$ Center for Computational Quantum Physics, Flatiron Institute, 162 Fifth avenue, New York, NY 10010, USA \\ ${ }^{6}$ Department of Quantum Matter Physics, University of Geneva, \\ 24 Quai Ernest-Ansermet, 1211 Geneva 4, Switzerland
}

(Dated: December 19, 2017)

\begin{abstract}
We investigate the interplay of spin-orbit coupling (SOC) and electronic correlations in $\mathrm{Sr}_{2} \mathrm{RuO}_{4}$ using dynamical mean-field theory. We find that SOC does not affect the correlation-induced renormalizations, which validates the Hund's metal picture of ruthenates even in the presence of the sizeable SOC relevant to these materials. Nonetheless, SOC is found to change significantly the electronic structure at k-points where a degeneracy applies in its absence. We explain why these two observations are consistent with one another and calculate the effects of SOC on the correlated electronic structure. The magnitude of these effects is found to depend on the energy of the quasiparticle state under consideration, leading us to introduce the notion of an energy-dependent quasiparticle spin-orbit coupling $\lambda^{*}(\omega)$. This notion is generally applicable to all materials in which both the spin-orbit coupling and electronic correlations are sizeable.
\end{abstract}

The effect of spin-orbit coupling (SOC) on the electronic properties of materials is a topic of broad current interest. In weakly correlated metals, SOC influences band-structure topology, and plays a key role for topological insulators and Weyl metals [1, 2]. In Mott insulators, SOC influences the atomic multiplet configurations and the magnetic exchange, which leads to rich spin-orbital physics [3 6]. SOC is also crucial for heavy-fermion compounds, where it selects the multiplet structure of the localized $f$-electrons [7, 8]. In contrast to these limiting cases, the effects of SOC in intermediate to strongly correlated metals have been less explored and are less understood.

A notable example [9 15] of such a metal is $\mathrm{Sr}_{2} \mathrm{RuO}_{4}$, which behaves as a strongly correlated Fermi liquid below $T_{\mathrm{FL}} \sim 25 \mathrm{~K}$, and undergoes a transition to an unconventional superconducting state below $1.5 \mathrm{~K}$ [16]. The role played by SOC in ruthenates raises an interesting puzzle. On the one hand, ruthenates have been successfully described [17] as 'Hund's metals', a family of compounds in which the electronic correlations are driven by Hund's coupling [18. Qualitative understanding and, importantly, quantitatively accurate results have been obtained [17, 19, 22] using dynamical mean-field theory (DMFT [23]). While these calculations did not take SOC into account, several physical properties were described satisfactorily including the measurements of the Knight shift and $1 / T_{1}$ from NMR, the frequency dependence of the self-energy [17, the optical conductivity [21] and the Seebeck coefficient 22 . In a broader context, direct evidence for the importance of Hund's coupling for the physics of ruthenates was recently obtained from photoemission experiments on insulating $\mathrm{Ca}_{2} \mathrm{RuO}_{4}[24$. One of the hallmarks of Hund's metals is a large degree of differ- entiation between the different orbitals (here forming the partially occupied $t_{2 g}$ subshell). Indeed, a much larger mass enhancement is observed for $d_{x y}$ than for $d_{x z, y z}$ in $\mathrm{Sr}_{2} \mathrm{RuO}_{4}$ [17, 25]. Because SOC promotes orbital mixing, such a differentiation would not be observed if SOC would dominate the physics. Another hallmark of Hund's metals 26-29] is that the temperature below which the orbital angular momentum no longer fluctuates is much higher ( $\gtrsim 1000 \mathrm{~K}$ in $\mathrm{Sr}_{2} \mathrm{RuO}_{4}[22$ ) than the corresponding temperature for spin degrees of freedom $\left(\sim T_{\mathrm{FL}}\right)$, pointing at a separation between spin and orbital degrees of freedom above $T_{\mathrm{FL}}$. All these remarks suggest that SOC might not crucially influence electronic correlations in $\mathrm{Sr}_{2} \mathrm{RuO}_{4}$.

On the other hand, the magnitude of the SOC $\lambda \simeq$ $0.1 \mathrm{eV}$ is much larger than low-energy scales such as $T_{\mathrm{FL}}$ and is not negligible in comparison to the Hund's rule coupling $J_{H} \approx 0.4 \mathrm{eV}$. Indeed, there is ample theoretical and experimental evidence that SOC does play an important role in $\mathrm{Sr}_{2} \mathrm{RuO}_{4}$. Band-structure calculations have demonstrated that it influences the shape of the Fermi surface (FS) in an important manner [30. Angular-resolved photoemission spectroscopy (ARPES) has revealed orbital mixing 31 and sizeable lifting of degeneracies 32 between bands. Recent DMFT calculations at the model level 33 and for $\mathrm{Sr}_{2} \mathrm{RuO}_{4} 34$ emphasized the importance of SOC, the latter work pointing at its enhancement by correlations.

The purpose of the present article is to resolve this apparent conflict. We analyse the renormalizations associated with the orbital-diagonal and orbital off-diagonal components of the self-energy and find striking differences between them. The former are frequencydependent ('dynamical') but essentially unaffected by 
Table I. Hopping amplitudes $t_{a b}^{\mathbf{R}}$ between orbitals $(a, b=$ $x y, x z, y z)$ along a lattice vector $\mathbf{R}$, entering the tight-binding hamiltonian $H_{0}$. We use $t=0.42, t_{1}=0.17, t_{2}=0.30, t_{3}=0.03$, and $t_{4}=0.04(\mathrm{eV})$ 37, and crystal field term $\epsilon_{\mathrm{CF}}\left(n_{x z}+n_{y z}\right)$ with $\epsilon_{\mathrm{CF}}=0.1 \mathrm{eV}$. Due to the Hund's rule coupling, the results are robust with respect to the changes of crystalfield. 38.

\begin{tabular}{lcccc}
\hline \hline & $x z, x z$ & $y z, y z$ & $x y, x y$ & $x z, y z$ \\
$t_{a b}^{ \pm 1,0,0}$ & $-t_{2}$ & $-t_{3}$ & $-t$ & 0 \\
$t_{a b}^{0, \pm 1,0}$ & $-t_{3}$ & $-t_{2}$ & $-t$ & 0 \\
$t_{a b}^{ \pm 1, \pm 1,0}$ & 0 & 0 & $-t_{1}$ & $-t_{4}$ \\
$t_{a b}^{ \pm 1, \mp 1,0}$ & 0 & 0 & $-t_{1}$ & $t_{4}$ \\
\hline \hline
\end{tabular}

SOC. In contrast, the latter are static (frequencyindependent) over a wide energy range, corresponding to a renormalization of the bare SOC by local interactions. This observation provides the solution to the above puzzle. The overall dynamical correlations, and in particular the correlation-induced orbitally-dependent renormalizations remain characteristic of Hund's metals, while the effect of the SOC is sizeable only close to quasi-momenta in the Brillouin zone where degeneracies (or quasi-degeneracies) are found for $\lambda=0$. The calculated electronic structure agrees well with photoemission experiments [31, 32]. We also provide analytical understanding of the effects of SOC close to degeneracy points. Importantly, because of the energy dependence of the many-body renormalizations, the splitting induced by SOC is found to depend on the energy of the states under consideration. This leads us to introduce the concept of an energy-dependent quasiparticle spin-orbit coupling, $\lambda^{*}(\omega)$.

Previous work on $\mathrm{Sr}_{2} \mathrm{RuO}_{4}$ disregarded the effects of SOC, with the exception of Ref. 34] who found that SOC is enhanced by the off-diagonal components of the selfenergy. However, the authors of Ref. [34] did not consider the properties of states away from the Fermi level and hence did not reveal the energy-dependence of the effect of SOC on quasiparticles, as described by $\lambda^{*}(\omega)$. This is crucial to understand the effect of SOC on the ARPES spectra of ruthenates 32 but also for other materials, such as iron-based superconductors 35, 36.

We describe $\mathrm{Sr}_{2} \mathrm{RuO}_{4}$ using the model Hamiltonian, $H=H_{0}+H_{\mathrm{ls}}+H_{\text {int }}$. $H_{0}$ is a TB hamiltonian with parameters specified in Table I] see also SM [38. In the basis having $m$ as a quantum number of $l_{z}\left(l_{z}\right.$ is the angular momentum operator projected on $t_{2 g}$ manifold), the SOC is described by:

$$
\begin{aligned}
H_{\mathrm{ls}}= & \frac{\lambda_{z}}{2} \sum_{m=-1}^{1} m\left(d_{m \uparrow}^{\dagger} d_{m \uparrow}-d_{m \downarrow}^{\dagger} d_{m \downarrow}\right) \\
& +\frac{\lambda_{x y}}{\sqrt{2}} \sum_{m=-1}^{0}\left(d_{m+1 \downarrow}^{\dagger} d_{m \uparrow}+d_{m \uparrow}^{\dagger} d_{m+1 \downarrow}\right)
\end{aligned}
$$

with $\lambda_{\mathrm{xy}}=\lambda_{\mathrm{z}}=\lambda=100 \mathrm{meV}$. (Distinct symbols $\lambda_{\mathrm{xy}}, \lambda_{\mathrm{z}}$ are introduced for later convenience). The Coulomb interactions $H_{\text {int }}$ within the $t_{2 g}$ manifold are described using the rotationally invariant Kanamori Hamiltonian $H_{\text {int }}=\left(U-3 J_{\mathrm{H}}\right) \hat{N}(\hat{N}-1) / 2-2 J_{\mathrm{H}} \vec{S}^{2}-J_{\mathrm{H}} \vec{L}^{2} / 2$ with $\hat{N}, \vec{S}, \vec{L}$ being the atomic charge, spin and angular momentum operators. We used $U=2.3 \mathrm{eV}$ and $J_{H}=0.4 \mathrm{eV}$, as established in previous work [17] and fixed the occupancy to $\langle\hat{N}\rangle=4$, as relevant to ruthenates. $H$ was solved in the framework of DMFT, using the hybridization expansion continuous-time quantum Monte Carlo (CTQMC) solver [39, as implemented in the TRIQS library [40, 41, with complex-valued imaginary time Green's functions, as required in general when handling SOC [38. The analytical continuation of Matsubara self energies to the real axis was performed using the stochastic analytical continuation [42. The temperature of simulations was $230 \mathrm{~K}$ for all the results described in the main text.

We first discuss the basic phenomenology emerging from the calculated electronic structure, as displayed on Fig. 1(a). The filled symbols indicate the location of the maxima of the spectral functions, obtained from DMFT including SOC, along the two directions $\Gamma-X$ and $\Gamma-M$ in the Brillouin zone. For comparison, the non-interacting band-structure from the TB+SOC Hamiltonian is shown with thin lines, as well as the ARPES data by Iwasawa et al [31]. The basic experimental observations are well reproduced by DMFT. We note in particular: (i) the strong quasiparticle mass renormalizations near Fermi
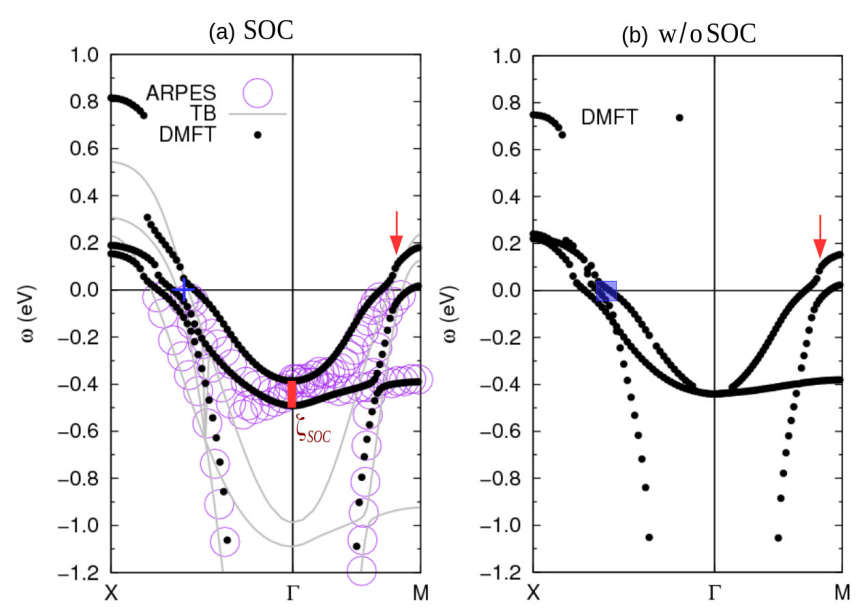

Figure 1. (a) DMFT+SOC quasiparticle dispersions along the X- $\Gamma-\mathrm{M}$ path (black dots). The ARPES measurements 31 ] are displayed as open circles, and the bands from $\mathrm{TB}+\mathrm{SOC}$ in the absence of interactions as light gray lines. The SOCinduced splitting at the $\Gamma$ point is indicated as a vertical bar. The cross at Fermi level along the $X-\Gamma$ path indicates the SOC-induced band splitting near the Fermi level. (b) Same as (a) but for DMFT without SOC. The square indicates the point where the $\beta$ and $\gamma$ sheets are nearly degenerate. The arrows indicate Fermi surface crossings along $\Gamma-\mathrm{M}$ at which the effect of SOC is small. 
(a) DMFT, SOC

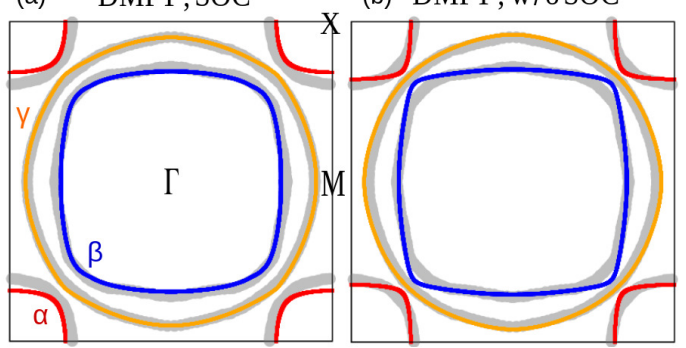

Figure 2. FSs of $\mathrm{Sr}_{2} \mathrm{RuO}_{4}$ in the DMFT with included SOC (a) and DMFT without SOC (b). The blue, orange, and red lines present $\beta, \gamma$, and $\alpha$ sheets. The FS from the ARPES experiment Ref. 45] is also reproduced (gray).

level and their marked orbital dependence [13, 17]: $\sim 4.0$ for $x y, \sim 3.1$ for $x z, y z$ [43. (ii) 'Unrenormalization' of the quasiparticle dispersion as the binding energy is increased, with effective velocities becoming closer to the bare ones. This effect is accompanied by waterfall structures 44 - an effect which has been observed in measurements of the optical conductivity [21] and also visible on Fig. 1 close to $\mathrm{X}$ point. (iii) Because of these unrenormalizations, the overall bandwidth of the $x z / y z$ quasiparticle band is about half of the non-interacting one, whereas that of the $x y$-band is approximately equal to the non-interacting one (despite the strong low-energy mass renormalizations). These unrenormalizations will play an important role also for the energy dependent effects of $\operatorname{SOC} \lambda^{*}(\omega)$.

A comparison with Fig. 1(b) (displaying our DMFT results without SOC, consistent with previous work [17. 21]) reveals that all the basic features (i-iii) are already correctly described without taking SOC into account. Nonetheless, the SOC has several important consequences that we now discuss. As apparent from Fig. 1 $(a, b)$, the effect of SOC is strongest at (near)degeneracy points. We emphasize in particular: (i) at the $\Gamma$-point and for a binding energy $\sim-0.5 \mathrm{eV}$, the lifting of degeneracy by $\zeta_{\mathrm{SOC}} \simeq 106 \mathrm{meV}$ between the two bands originating from the $x z, y z$ orbitals (signalled by a red bar on Fig. 1a), and (ii) along the $\Gamma-X$ direction, at the Fermi level, the $\beta$ and $\gamma$ sheets of the Fermi surface almost touch when SOC is neglected (as indicated by the blue square on panel (b) of Fig. 11. The inclusion of SOC lifts this degeneracy by an amount $\zeta_{\text {SOC }}^{\prime} \simeq 104 \mathrm{meV}$ (blue cross in panel (a)). Correspondingly, the Fermi momenta are shifted by an amount $\pm \delta_{k}$ and, as shown below, the Fermi velocities of the two sheets become equal. These effects are typical of level repulsion. Away from the neardegeneracies the effect of SOC is smaller: the dispersions along $\Gamma-M$ and the corresponding Fermi surface crossings (around the red arrows on Fig 1) are affected less. In particular, the Fermi velocities and the magnitude of the renormalizations are orbital/sheet-dependent.
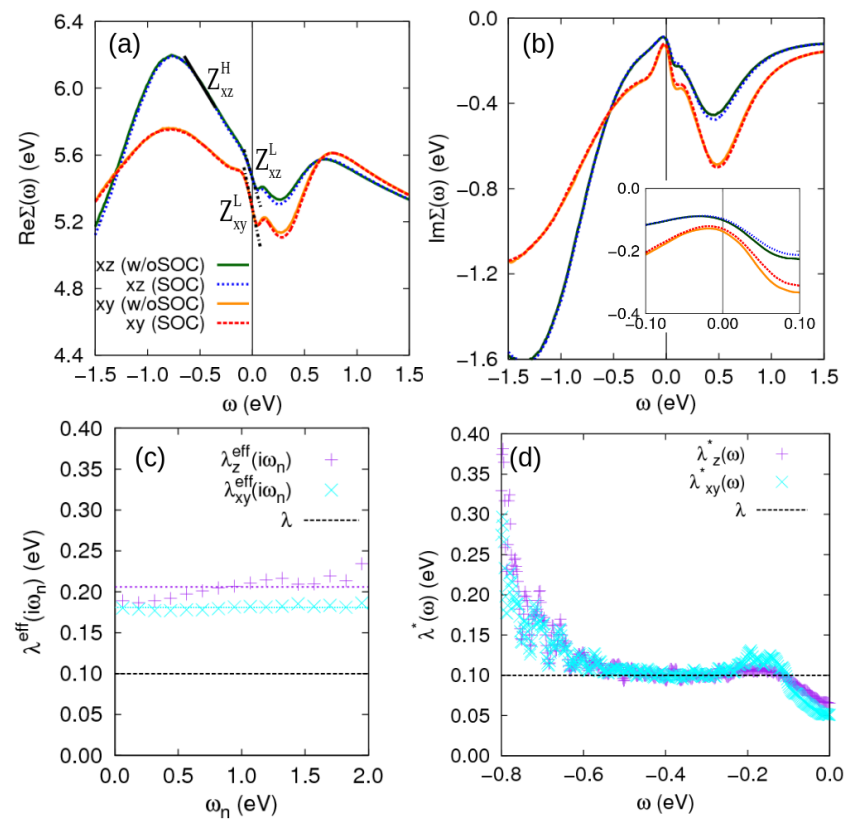

Figure 3. Real (a) and imaginary (b) parts of the real-frequency orbital-diagonal components of the self-energy (with SOC dashed, without SOC plain). Two linear fits const $+\omega(1-1 / Z)$ are also displayed in (a), corresponding to low-frequency (dashed line, slope set by $Z_{a}^{\mathrm{L}}$ ) and higher frequency around $\omega=-0.5 \mathrm{eV}$ (plain line, slope set by $Z_{a}^{\mathrm{H}}$ ). (c): Off-diagonal components of the Matsubara self-energy, presented as an effective renormalization of SOC (see text). Note the weak frequency dependence. The straight horizontal lines denote average values over the range $[0,2] \mathrm{eV}$ in comparison to the bare value $\lambda=0.1 \mathrm{eV}$. (d) Energy dependent quasiparticle SOC $\lambda_{z}^{*}(\omega)$ and $\lambda_{x y}^{*}(\omega)$ (see text).

The calculated FS including SOC is displayed on Fig. 2(a) and compared to the measured one [46]. The SOC modifies the Fermi surface, leading to an inflation of the $\gamma$-sheet. This inflation is not due to a change in orbital populations, which remain basically unchanged from their $\lambda=0$ values 0.64 and 0.68 (per spin) for $x y$ and $x z$, respectively. Instead, it is driven by the admixture of $x z / y z$ orbital character into the $\gamma$ sheet. The fact that orbital polarization in a DMFT calculation with SOC remains similar to the one found without taking SOC into account is another demonstration that the Hund's rule coupling physics is not affected by SOC. 38

In order to understand why the overall effect of correlations are unchanged by SOC while the fermiology close to degeneracy points is affected significantly, we examine the DMFT self-energies. The orbital-diagonal components are displayed in panels $(a, b)$ of Fig. 3 in the presence and absence of SOC, respectively. Strikingly, there is very little difference between the two calculations. All the key features (the linear low-frequency behaviour of the real parts $\propto\left(1-1 / Z_{a}\right) \omega$, followed by a kink at $\sim-50$ $\mathrm{meV}$, the features at positive energies, the frequency dependence of the imaginary part/scattering rate) are not 
only found to be similar but practically the same. Such insensitivity of electronic correlations to the SOC can be rationalized in terms of the high energy scale below which orbital fluctuations are screened, characteristic of Hund's metals 22, 47.

The effect of SOC on electronic correlations becomes apparent only when looking at the off-diagonal components of the self-energies $\Sigma_{x z ; x y}$ and $\Sigma_{x z ; y z}$. These vanish by symmetry for $\lambda=0$ but acquire a finite value once SOC is included. On Fig. 3(c) we display, as a function of Matsubara frequency $\omega_{n}$, the quantities [48]: $\lambda_{z}^{\mathrm{eff}}\left(i \omega_{n}\right)=\lambda_{\mathrm{SOC}}+2 \operatorname{Im} \Sigma_{x z \uparrow, y z \uparrow}\left(i \omega_{n}\right), \lambda_{x y}^{\mathrm{eff}}\left(i \omega_{n}\right)=\lambda_{\mathrm{SOC}}-$ $2 \operatorname{Im} \Sigma_{x z \uparrow, x y \downarrow}\left(i \omega_{n}\right)$. As seen there, the off-diagonal components are frequency-independent over a wide frequency range (exceeding $\sim 2 \mathrm{eV}$ ), hence they can be considered as additional single-particle terms that add-up to the bare ones [34, yielding $\lambda_{z}^{\mathrm{eff}}=206 \mathrm{meV}, \lambda_{x y}^{\mathrm{eff}}=181 \mathrm{meV}$, enhanced by about a factor of two over the bare value $\lambda=0.1 \mathrm{eV}$.

We are now in a position to perform a more quantitative analysis of the effects of SOC. Let us focus first on quasiparticle dispersions, which are the solutions $\omega(k)$ of:

$$
\operatorname{det}\left[(\omega+\mu) \delta_{\underline{a}, \underline{b}}-H^{0}(k)_{\underline{a}, \underline{b}}-\operatorname{Re} \Sigma(\omega)_{a} \delta_{\underline{a}, \underline{b}}-\hat{\lambda}_{\underline{a}, \underline{b}}^{\mathrm{eff}}\right]=0
$$

in which here $\underline{a}, \underline{b}$ are indices for the six possible spinorbital combinations $(a \sigma)$. Close to the Fermi level, this equation can be simplified by inserting the linear frequency dependence of the self-energy $\operatorname{Re} \Sigma_{a}=\Sigma_{a}(0)+$ $\omega\left(1-1 / Z_{a}\right)+\cdots$ and linearizing in the momentumdependence close to the FS. Considering for example the $\Gamma$ - $X$ direction for which the $\beta$ and $\gamma$ bands are almost degenerate close to the FS in the absence of SOC (Fig 11), and considering the $\alpha$-band to be lower in energy, an analytical solution to Eq. (2) can be obtained [38]. This leads to the following expression for the SOC-induced splitting in momentum $\pm \delta_{k}$ and energy $\zeta_{\text {SOC }}^{\prime}$, and for the renormalized quasiparticle velocities:

$$
\begin{gathered}
\delta_{k}=\frac{\lambda_{x y}^{\mathrm{eff}}}{2 \sqrt{v_{\beta} v_{\gamma}}}=\frac{\sqrt{Z_{x z} Z_{x y}} \lambda_{x y}^{\mathrm{eff}}}{2 \sqrt{v_{\beta}^{*} v_{\gamma}^{*}}}, v^{*}=2 \frac{v_{\beta}^{*} v_{\gamma}^{*}}{v_{\beta}^{*}+v_{\gamma}^{*}} \\
\zeta_{\mathrm{SOC}}^{\prime}=2 v^{*} \delta_{k}=2 \frac{\sqrt{v_{\beta}^{*} v_{\gamma}^{*}}}{v_{\beta}^{*}+v_{\gamma}^{*}} \sqrt{Z_{x y} Z_{x z}} \lambda_{x y}^{\mathrm{eff}}=\frac{v^{*}}{\sqrt{v_{\beta}^{*} v_{\gamma}^{*}}} \lambda_{x y}^{*}(0)
\end{gathered}
$$

In this expression, $v_{\beta}, v_{\gamma}$ are the bare TB Fermi velocities in the absence of SOC and interactions for the $\beta$ and $\gamma$ sheets, and $v_{\beta}^{*}=Z_{x z} v_{\beta}, v_{\gamma}^{*}=Z_{x y} v_{\gamma}$ are the renormalized quasiparticle velocities in the presence of interactions but without SOC. We have introduced energy-dependent quasiparticle spin-orbit couplings, defined by:

$$
\lambda_{z}^{*}(\omega)=Z_{x z}(\omega) \lambda_{z}^{\mathrm{eff}}, \lambda_{x y}^{*}=\sqrt{Z_{x y}(\omega) Z_{x z}(\omega)} \lambda_{x y}^{\mathrm{eff}}
$$

The lifting of degeneracy by SOC equalizes the velocities for each sheet, hence suppressing the orbital/sheet differentiation at this specific k-point, an effect clearly visible on Fig. 1 (a), which deserves to be resolved from high
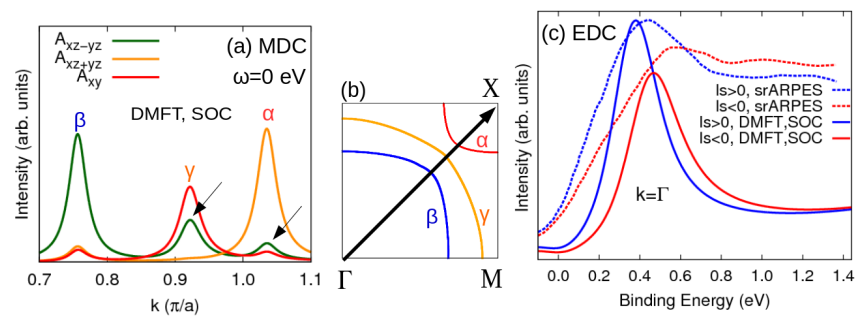

Figure 4. (a) Momentum distribution curves (MDC) at zero energy $\omega=0$ along the $\Gamma$-X path, projected on the $x y, x z-y z$ and $x z+y z$ orbital components. The arrows emphasize the non-zero contribution of the $x z-y z$ orbital to the $\gamma$ and $\alpha$ bands. (c) Energy distribution curves (EDC) at the $\Gamma$-point, for states with spin parallel $(l s>0)$ and anti-parallel $(l s<0)$ to the orbital moment, compared to spin-resolved ARPES results 32. A Gaussian broadening of $0.05 \mathrm{eV}$ was used.

resolution ARPES experiments. We find $\delta_{k} \simeq 0.087 \pi / a$ and $\zeta_{\text {SOC }}^{\prime} \simeq 104 \mathrm{meV}$. Away from degeneracy points (e.g. for FS crossings along $\Gamma-\mathrm{M})$, the SOC can be treated perturbatively and only modifies weakly the Fermi momenta and quasiparticle velocities by an amount of order $\left(\lambda^{\text {eff }}\right)^{2} / \Delta \varepsilon$, with $\Delta \varepsilon$ the quasiparticle energy separation between the considered band and the one closest to it in energy. Note also that the effective coupling $\lambda_{x y}^{*}(0)$ which enters $\zeta_{\text {SOC }}^{\prime}$ is suppressed by the renormalization factor $\sqrt{Z_{x y} Z_{x z}} \approx 1 / 4$ as compared to $\lambda^{\text {eff }}$. The electronic correlations thus, on one hand, increase the magnitude of the SOC through the orbital off-diagonal self-energy but, on the other hand, suppress it due to the quasiparticle renormalization factor.

Further insight into the orbital content of each FS sheet along the $\Gamma$-X direction can be obtained by looking at the momentum-distribution curves (MDCs), which are displayed on Fig. 4(a) by using a projection onto the $x y$, $x z-y z$ and $x z+y z$ orbital components. We observe that the $\gamma$-sheet crossing has almost equal contributions from $x y$ and $x z-y z$ orbital components, as previously discussed from electronic structure calculations [30] and ARPES experiments [31. The fact that all sheets have a non-vanishing $x z-y z$ component (odd under mirrorplane $x \leftrightarrow y$ symmetry) is consistent with the ARPES results of Ref. [31 for sigma-polarized light.

The quasiparticle equation (2) can also be used to discuss the SOC-induced $\Gamma$-point splitting between the $x z$ and $y z$-dominated bands at a binding energy $\omega_{\Gamma} \sim-0.5 \mathrm{eV}$. The key difference here (in contrast to the energy splitting at the FS, $\zeta_{\text {SOC }}^{\prime}$ ) is that the quasiparticle renormalization appropriate to this higher energy must be used (Fig. 3), which is given by $\left[\left.\left(1-d \operatorname{Re} \Sigma_{x z} / d \omega\right)\right|_{\omega=\omega_{\Gamma}}\right]^{-1}=Z_{x z}\left(\omega_{\Gamma}\right)=0.49$, different (and larger) from the low-energy values $Z_{x z}=Z_{y z} \simeq$ 0.32. As a result, the SOC-induced $\Gamma$-point splitting reads

$$
\zeta_{\mathrm{SOC}}=Z_{x z}\left(\omega_{\Gamma}\right) \lambda_{z}^{\mathrm{eff}}=\lambda_{z}^{*}\left(\omega_{\Gamma}\right)
$$


The energy dependence of the quasiparticle renormalization $Z(\omega)$ thus causes the effects of the SOC also to be energy dependent, and given by $\lambda^{*}(\omega)$. This energydependent SOC acting on quasiparticles states is plotted on Fig. 3(d).

We note that at $\omega_{\Gamma}$ there is a compensation between the correlation-induced enhancement $\lambda_{z}^{\mathrm{eff}} \simeq 2 \lambda$ and the renormalization by $Z_{x z}^{\mathrm{H}} \simeq 1 / 2$, so that we obtain $\zeta_{\mathrm{SOC}} \simeq$ $106 \mathrm{meV}$ to be quite close to the bare $\lambda$. It is only due to this accidental cancellation that the LDA [30] correctly predicted the observations of spin-resolved ARPES 32. Last, we turn to a closer comparison of our result to this experiment that selectively probed electrons with orbital angular momentum parallel $(l s>0)$ and anti-parallel $(l s<0)$ to the electron spin 32 . Hence, we display in Fig. 4(c) the calculated $l s$-resolved spectral functions at the $\Gamma$ point. The location of the maxima of the two peaks, their splitting $\zeta_{\mathrm{SOC}} \simeq 106 \mathrm{meV}$ and the lower intensity of the higher binding energy $l s<0$ peak are well reproduced.

In summary, there are three qualitative take-home messages from our work. First, because the SOC is smaller than the orbital coherence scale, it does not affect the dynamical properties of $\mathrm{Sr}_{2} \mathrm{RuO}_{4}$, and electronic correlations are characteristic of a Hund's metal. This validates previous theoretical work that neglected SOC [17, 19, 22, 49,52. Second, the SOC does change significantly the electronic structure at k-points where a degeneracy is found in the absence of SOC. Thirdly, when evaluating these effects, it is crucial to take into account both the static renormalization of the SOC by correlations, and the quasiparticle renormalization factors taken in the appropriate energy range, leading to the notion of an energy-dependent quasiparticle SOC. These observations are important for the ongoing discussion of the superconducting order parameter of $\mathrm{Sr}_{2} \mathrm{RuO}_{4}$ where SOC plays a key role [11, [53, and are of general relevance to a broad class of materials involving SOC and electronic correlations.

We are grateful to M. Aichhorn, F. Baumberger, G. Kraberger and A. Tamai for useful discussions and to the CPHT computer support team. This work was supported by the European Research Council grants ERC-319286-QMAC (M.K., A.G.) and ERC-278472MottMetals (O.P.), and by the Swiss National Science Foundation (NCCR MARVEL). The Flatiron Institute is supported by the Simons Foundation. J.M. acknowledges support by the Slovenian Research Agency (ARRS) under Program P1-0044.

\footnotetext{
*arix.minjae.kim@gmail.com

[1] M. Z. Hasan and C. L. Kane, Reviews of Modern Physics 82, 3045 (2010).
}

[2] X. Wan, A. M. Turner, A. Vishwanath, and S. Y. Savrasov, Physical Review B 83, 205101 (2011).

[3] K. Yosida, Theory of Magnetism (Springer Series in Solid-State Sciences) (Springer, 2001).

[4] D. I. Khomskii, Transition Metal Compounds (Cambridge University Press, 2014).

[5] G. Jackeli and G. Khaliullin, Physical Review Letters 102, 017205 (2009).

[6] G. Khaliullin, Physical Review Letters 111, 197201 (2013).

[7] G. R. Stewart, Rev. Mod. Phys. 56, 755 (1984)

[8] M. Dzero, J. Xia, V. Galitski, and P. Coleman, Annual Review of Condensed Matter Physics 7, 249 (2016).

[9] M. Anwar, S. Lee, R. Ishiguro, Y. Sugimoto, Y. Tano, S. Kang, Y. Shin, S. Yonezawa, D. Manske, H. Takayanagi, et al., Nature communications 7 (2016).

[10] K. Nelson, Z. Mao, Y. Maeno, and Y. Liu, Science 306, 1151 (2004).

[11] B. Kim, S. Khmelevskyi, I. Mazin, D. Agterberg, and C. Franchini, npj Quantum Materials (2017).

[12] A. P. Mackenzie, T. Scaffidi, C. W. Hicks, and Y. Maeno, npj Quantum Materials (2017).

[13] B. Burganov, C. Adamo, A. Mulder, M. Uchida, P. King, J. Harter, D. Shai, A. Gibbs, A. Mackenzie, R. Uecker, et al., Physical Review Letters 116, 197003 (2016).

[14] C. W. Hicks, D. O. Brodsky, E. A. Yelland, A. S. Gibbs, J. A. Bruin, M. E. Barber, S. D. Edkins, K. Nishimura, S. Yonezawa, Y. Maeno, et al., Science 344, 283 (2014).

[15] A. Steppke, L. Zhao, M. E. Barber, T. Scaffidi, F. Jerzembeck, H. Rosner, A. S. Gibbs, Y. Maeno, S. H. Simon, A. P. Mackenzie, et al., Science 355, eaaf9398 (2017)

[16] A. P. Mackenzie and Y. Maeno, Reviews of Modern Physics 75, 657 (2003)

[17] J. Mravlje, M. Aichhorn, T. Miyake, K. Haule, G. Kotliar, and A. Georges, Physical Review Letters 106, 096401 (2011).

[18] A. Georges, L. de' Medici, and J. Mravlje, Annual Reviews of Condensed Matter Physics 4, 137 (2013), http://arxiv.org/abs/1207.3033.

[19] H. T. Dang, J. Mravlje, A. Georges, and A. J. Millis, Physical Review Letters 115, 107003 (2015).

[20] H. T. Dang, J. Mravlje, A. Georges, and A. J. Millis, Physical Review B 91, 195149 (2015).

[21] D. Stricker, J. Mravlje, C. Berthod, R. Fittipaldi, A. Vecchione, A. Georges, and D. van der Marel, Phys. Rev. Lett. 113, 087404 (2014)

[22] J. Mravlje and A. Georges, Phys. Rev. Lett. 117, 036401 (2016).

[23] A. Georges, G. Kotliar, W. Krauth, and M. J. Rozenberg, Rev. Mod. Phys. 68, 13 (1996).

[24] D. Sutter, C. Fatuzzo, S. Moser, M. Kim, R. Fittipaldi, A. Vecchione, V. Granata, Y. Sassa, F. Cossalter, G. Gatti, et al., Natue Communications 8, 15176 (2017).

[25] A. P. Mackenzie and Y. Maeno, Rev. Mod. Phys. 75, 657 (2003).

[26] Z. P. Yin, K. Haule, and G. Kotliar, Phys. Rev. B 86, $195141(2012)$

[27] C. Aron and G. Kotliar, Phys. Rev. B 91, 041110 (2015)

[28] K. Stadler, Z. Yin, J. von Delft, G. Kotliar, and A. Weichselbaum, Physical Review Letters 115, 136401 (2015).

[29] A. Horvat, R. Žitko, and J. Mravlje, Phys. Rev. B 94, $165140(2016)$

[30] M. Haverkort, I. Elfimov, L. Tjeng, G. Sawatzky, and 
A. Damascelli, Physical Review Letters 101, 026406 (2008).

[31] H. Iwasawa, Y. Yoshida, I. Hase, S. Koikegami, H. Hayashi, J. Jiang, K. Shimada, H. Namatame, M. Taniguchi, and Y. Aiura, Physical Review Letters 105, 226406 (2010).

[32] C. Veenstra, Z.-H. Zhu, M. Raichle, B. Ludbrook, A. Nicolaou, B. Slomski, G. Landolt, S. Kittaka, Y. Maeno, J. Dil, et al., Physical Review Letters 112, $127002(2014)$.

[33] A. J. Kim, H. O. Jeschke, P. Werner, and R. Valentí, Phys. Rev. Lett. 118, 086401 (2017).

[34] G. Zhang, E. Gorelov, E. Sarvestani, and E. Pavarini, Physical Review Letters 116, 106402 (2016).

[35] S. V. Borisenko, D. V. Evtushinsky, Z.-H. Liu, I. Morozov, R. Kappenberger, S. Wurmehl, B. BÃijchner, A. N. Yaresko, T. K. Kim, M. Hoesch, T. Wolf, and N. D. Zhigadlo, Nat. Phys. 12, 311 (2016).

[36] R. Day, G. Levy, M. Michiardi, M. Zonno, F. Ji, E. Razzoli, F. Boschini, S. Chi, R. Liang, P. Das, et al., arXiv preprint arXiv:1711.06686 (2017).

[37] S. Okamoto and A. J. Millis, Physical Review B 70, 195120 (2004).

[38] See the Supplemental Material for (i) the insensitivity of the crystal-field independent electronic structures, (ii) discussion of our implementation of the solver, (iii) SOC effects on the momentum distribution curve at $\omega=0$, (iv) analytical discussion of SOC induced splitting of quasiparticle bands, (v) Matsubara self-energies and the discussion of the temperature dependence, and (vi) plots of FS for cases without SOC and without correlations.

[39] E. Gull, A. J. Millis, A. I. Lichtenstein, A. N. Rubtsov, M. Troyer, and P. Werner, Rev. Mod. Phys. 83, 349 (2011).
[40] O. Parcollet, M. Ferrero, T. Ayral, H. Hafermann, I. Krivenko, L. Messio, and P. Seth, Computer Physics Communications 196, 398 (2015)

[41] P. Seth, I. Krivenko, M. Ferrero, and O. Parcollet, Computer Physics Communications 200, 274 (2016)

[42] K. Beach, arXiv preprint cond-mat/0403055 (2004).

[43] The renormalizations depend on temperature and increases towards the measured ones when the temperature of the simulation is reduced. [54].

[44] H. Iwasawa, Y. Yoshida, I. Hase, K. Shimada, H. Namatame, M. Taniguchi, and Y. Aiura, Physical Review Letters 109, 066404 (2012)

[45] A. Damascelli, D. Lu, K. Shen, N. Armitage, F. Ronning, D. Feng, C. Kim, Z.-X. Shen, T. Kimura, Y. Tokura, et al., Physical Review Letters 85, 5194 (2000).

[46] Longer range hoppings and out-of-layer tunneling terms would improve quantitative agreement with experiment.

[47] A. Horvat, J. Mravlje, et al., Physical Review B 96, 085122 (2017)

[48] Equivalently, $\lambda_{x y}^{\text {eff }}$ can be defined from $\operatorname{Re} \Sigma_{y z \uparrow, x y \downarrow}$, which is identical to $\operatorname{Im} \Sigma_{x z \uparrow, x y \downarrow}$ by symmetry.

[49] M. Kim and B. Min, Physical Review B 91, 205116 (2015).

[50] Q. Han, H. T. Dang, and A. Millis, Physical Review B 93, 155103 (2016).

[51] N. Dasari, S. S. Yamijala, M. Jain, T. S. Dasgupta, J. Moreno, M. Jarrell, and N. Vidhyadhiraja, Physical Review B 94, 085143 (2016).

[52] X. Deng, K. Haule, and G. Kotliar, Physical Review Letters 116, 256401 (2016).

[53] S. Cobo, F. Ahn, I. Eremin, and A. Akbari, Phys. Rev. B 94, 224507 (2016)

[54] X. Deng, J. Mravlje, R. Žitko, M. Ferrero, G. Kotliar, and A. Georges, Phys. Rev. Lett. 110, 086401 (2013) 